\title{
Butorphanol protects PC12 cells against OGD/R-induced inflammation and apoptosis
}

\author{
ZIJING YANG, LI WANG, YINGJUN HU and FEIXIANG WANG \\ Department of Anesthesiology, The First People's Hospital of Tonglu, Tonglu, Zhejiang 311500, P.R. China
}

Received January 2, 2020; Accepted May 19, 2020

DOI: $10.3892 / \mathrm{mmr} .2020 .11290$

\begin{abstract}
The aim of the present study was to examine the effects of butorphanol on neural injury in an oxygen glucose deprivation/reoxygenation (OGD/R) model using PC12 cells, and to investigate whether mitochondrial apoptosis was involved in these effects. To establish the OGD/R model, PC12 cells were cultured under hypoxia and low glucose conditions. Expression levels of inflammatory cytokines were evaluated by detecting the levels of tumor necrosis factor- $\alpha$, interleukin (IL)-1 $\beta$, IL-6 and monocyte chemoattractant protein-1. Oxidative stress was evaluated by measuring the levels of reactive oxygen species, lactate dehydrogenase activity and myeloperoxidase concentration. Apoptosis, protein expression and cell viability were determined by flow cytometry, western blotting and by using a Cell Counting Kit-8, respectively. Compared with the control group, cell viability, expression of inflammatory factors and oxidative stress were all decreased in the OGD/R group. All the above changes could be mitigated by treatment with butorphanol. In addition, butorphanol treatment resulted in a significant upregulation of Bax, and downregulation of Bcl-2, activated caspase-3, caspase- 9 and poly ADP-ribose polymerase, increased the expression of $\mathrm{X}$-linked inhibitor of apoptosis protein and enhanced ATP activity. To conclude, these results suggested that the protective effects of butorphanol are associated with the inhibition of OGD/R-induced inflammation and apoptosis injury, and may be partially associated with the inhibition of mitochondrial apoptosis.
\end{abstract}

\section{Introduction}

Cerebrovascular disease is a common disease that poses a significant threat to human health, particularly in individuals $>50$ years old, and ischemic cerebrovascular disease accounts

Correspondence to: Dr Zijing Yang, Department of Anesthesiology, The First People's Hospital of Tonglu, 338 Xuesheng Road, South Street, Tonglu, Zhejiang 311500, P.R. China

E-mail: zi_jing71@163.com

Key words: butorphanol, oxygen glucose deprivation/reoxygenation, neural injury, mitochondrial apoptosis, oxidative stress for $60-80 \%$ of cases $(1,2)$. Ischemia-reperfusion injury is an important pathophysiological process in the pathogenesis of ischemic cerebrovascular disease $(3,4)$. Under ischemic conditions, the brain cannot provide the energy required for biosynthesis and positive ions transformation of neurotransmitters, and for proper function of enzymes and cell membrane structures, resulting in excessive release and difficulty in the reuptake of excitatory neurotransmitters, cytoskeleton cleavage and antioxidant damage, resulting in neurological damage (5-7). The energy changes caused by mitochondrial dysfunction can directly induce neuronal apoptosis or necrosis. A large number of pro-apoptotic molecules that are stored in the mitochondria and do not exhibit their effects under physiological conditions; however, these molecules result in apoptosis through a series of physiological changes induced by a variety of apoptosis signals, which also suggests mitochondrial apoptosis serves a key role in cell death induced by hypoxic-ischemic brain damage (8-10).

Butorphanol is a synthetic selective opioid receptor antagonist with substantial analgesic effects (11). The mechanisms underlying its function is dependent on the dual pharmacological functions of metabolite activated $\kappa$-opioid receptor and $\mu$-receptor $(12,13)$. Butorphanol can be administered in a variety of ways, including intravenous injection, muscular injection and nasal sprays. Previous studies have demonstrated that butorphanol exhibits potential for a wide range of clinical applications, including the treatment of opioid-induced pruritus (14). Additionally, butorphanol can be used as an adjuvant therapy in opioid-dependent patients (15). Huang et al (16) reported that butorphanol could attenuate myocardial ischemia reperfusion injury through inhibiting mitochondria-mediated apoptosis in mice. Zhang et al (17) demonstrated that fentanyl combined with butorphanol protected against myocardial ischemia-reperfusion injury via activation of nuclear factor erythroid 2-related factor 2 -antioxidant response elements signaling. In addition, in vivo experiments reported that butorphanol exhibits a significant protective effect on myocardial ischemia-reperfusion injury in rats, reducing ischemic myocardial ischemia and infarct size, reducing the levels of pro-inflammatory cytokines or increasing the production of anti-inflammatory cytokines to reduce myocardial reperfusion-induced myocardial injury, and it also reduced myocardial ischemic injury by inhibiting mitochondrial-mediated apoptosis (18). However, the neuroprotective effects of butorphanol on oxygen glucose 
deprivation/reoxygenation (OGD/R) injury of nerve cells has not been determined previously, to the best of our knowledge.

PC12 cells are derived from a pheochromocytoma of the rat adrenal medulla. Use of this cell line has several advantages, including high purity, rapid growth and reproduction. It is a widely used cell line for studying the function, differentiation, apoptosis and potential molecular mechanism of nerve cells $(19,20)$. In the present study, the effects of butorphanol on hypoxic-induced ischemia-reperfusion injury of PC12 cells were investigated. In addition, the involvement of mitochondrial apoptosis in the butorphanol-mediated effects was assessed.

\section{Materials and methods}

Reagents and chemicals. Butorphanol Tartrate Injection was purchased from Jiangsu Hengrui Medicine Co., Ltd. PC12 cells were obtained from the American Type Culture Collection. DMEM, Earle's balanced salt solution (EBSS), FBS, $0.25 \%$ trypsin and PBS were obtained from Gibco (Thermo Fisher Scientific, Inc.). Cell Counting Kit-8 (CCK-8) was obtained from Dojindo Molecular Technologies, Inc. Tumor necrosis factor (TNF)- $\alpha$ (cat. no. PT512), interleukin (IL)-6 (cat. no. PI326), IL-1 $\beta$ (cat. no. PI301) and monocyte chemotactic protein-1 (MCP-1; cat. no. PC125) detection kits were provided by Beyotime Institute of Biotechnology. Reactive oxygen species (ROS cat. no. AAT-16053), lactate dehydrogenase (LDH; cat. no. 10008882-96), myeloperoxidase (MPO; cat. no. HK105-01) and ATP (cat. no. 701004-4) detection kits were provided by AmyJet Scientific, Inc. Annexin V-FITC reagents were purchased from BD Biosciences. PVDF membranes were provided by EMD Millipore. DAPI reagents (cat. no. C1002) and FITC-conjugated goat anti-rabbit IgG antibodies (cat. no. A0562) were purchased from Beyotime Biotechnology Co., Ltd. Primary antibodies against Bcl-2 (cat. no. 2875), Bax (cat. no. 14796), cleaved-poly ADP-ribose polymerase (PARP) (cat. no. 9532), caspase-3 (cat. no. 14220), caspase-9 (1:1,000; cat. no. 9508), X-linked inhibitor of apoptosis protein (XIAP; cat. no. 14334), and $\beta$-actin (cat. no. 3700) were all from Cell Signaling Technology, Inc. The horseradish peroxidase (HRP)-conjugated goat anti-rabbit secondary antibody (cat. no. bs-0296G-HRP) was purchased from BIOSS. The FITC-conjugated goat anti-rabbit IgG antibody (cat. no. sc-2359-FITC) was obtained from Santa Cruz Biotechnology. All the other chemicals and reagents were of analytical grade.

Cell culture. PC12 cells were cultured in a glass culture flask filled with DMEM containing $10 \%$ FBS and placed in a $\mathrm{CO}_{2}$ incubator $\left(95 \% \mathrm{O}_{2}\right.$ and $\left.5 \% \mathrm{CO}_{2}\right)$ at $37^{\circ} \mathrm{C}$. The medium was changed every $48 \mathrm{~h}$ and cells were subcultured at $80-90 \%$ confluency. Subsequently, cells were subcultivated. Cells in the logarithmic growth phase were used for subsequent experiments.

$O G D / R$ model. DMEM was replaced by glucose-free EBSS solution, and $2 \times 10^{5}$ cells $/ \mathrm{ml}$ PC12 cells were cultured in the three-gas incubator $\left(94 \% \mathrm{~N}_{2}, 5 \% \mathrm{CO}_{2}\right.$ and $\left.1 \% \mathrm{O}_{2}\right)$ at $37^{\circ} \mathrm{C}$ for $2 \mathrm{~h}$. Subsequently, the medium was replaced with DMEM and cells were incubated in the $\mathrm{CO}_{2}$ incubator $\left(95 \% \mathrm{O}_{2}\right.$ and
$5 \% \mathrm{CO}_{2}$ ) at $37^{\circ} \mathrm{C}$ for $24 \mathrm{~h}$. Control cells were routinely cultured under normoxic conditions.

Experimental groups. To determine a suitable dose of butorphanol for treating PC12 cells, cells were cultured with gradually increasing concentrations $(0-8 \mu \mathrm{M})$ of butorphanol at $37^{\circ} \mathrm{C}$ for $24 \mathrm{~h}$. Cell viability was analyzed using a CCK-8 assay according to the manufacturer's instructions, and three doses of butorphanol $(1,2$ and $4 \mu \mathrm{M})$ were selected for the subsequent experiment. PC12 cells were divided into five groups in the study: i) Control group; ii) $\mathrm{OGD} / \mathrm{R}$ group; iii) $\mathrm{OGD} / \mathrm{R}+1 \mu \mathrm{M}$ butorphanol group; iv) $\mathrm{OGD} / \mathrm{R}+2 \mu \mathrm{M}$ butorphanol group; and v) $\mathrm{OGD} / \mathrm{R}+4 \mu \mathrm{M}$ butorphanol group.

CCK-8 assay. Cell viability was detected using a CCK-8 assay according to the manufacturer's protocol. PC12 cells were seeded into 96 -well plates at a density of $5 \times 10^{3}$ cells/well and incubated at $37^{\circ} \mathrm{C}$ for $24 \mathrm{~h}$. CCK-8 solution $(20 \mu \mathrm{l})$ was added to each well followed by continuous incubation at $37^{\circ} \mathrm{C}$ for $2 \mathrm{~h}$. Absorbance was measured at a wavelength of $450 \mathrm{~nm}$ and cell activity was expressed as the ratio of the measured wavelength to the control wavelength.

ELISA assay. The supernatant of the PC12 cells was collected and centrifuged at $1,000 \mathrm{x}$ for $20 \mathrm{~min}$ at $4^{\circ} \mathrm{C}$ to remove any impurities and cell fragments. The levels of TNF- $\alpha$, IL-1 $\beta$, IL-6 and MCP-1 were detected according to the manufacturer's protocol. The standard curve was drawn with the standard concentration as abscissa and the absorbance at $450 \mathrm{~nm}$ wavelength as the ordinate values. The actual concentrations of samples were obtained according to the absorbance value.

Detection of oxidative stress. Oxidative stress was measured based on ROS, LDH and MPO activity using the corresponding kits. When determining ROS content, the time between loading the probe and measuring should be shortened as much as possible, to reduce various possible errors. LDH activity $(\mathrm{mU} / \mathrm{ml})$ was calculated as follows: (absorbance of sample - absorbance of background blank control)/(absorbance of standard - absorbance of standard blank) $\mathrm{x}$ concentration of standard substance $(\mathrm{mU} / \mathrm{ml})$.

Flow cytometry analysis. PC12 cells were digested with trypsin and resuspended to a density of $5 \times 10^{5}-1 \times 10^{6}$ cells $/ \mathrm{ml}$. The digested cells were centrifuged at $1,000 \mathrm{x}$ g for $10 \mathrm{~min}$ at $4^{\circ} \mathrm{C}$, and the supernatant was discarded. After adding $1 \mathrm{ml}$ PBS, the above steps were repeated three times, and cells were resuspended in $200 \mu \mathrm{l}$ buffer solution. A total of $10 \mu \mathrm{M}$ Annexin V-FITC was added to the cells and incubated at room temperature without light for $15 \mathrm{~min}$, and then $300 \mu \mathrm{l}$ buffer and $5 \mu \mathrm{l}$ propidium iodide were added. Apoptosis was detected using flow cytometry (FC 5000; BD Biosciences) within $1 \mathrm{~h}$, and data were analyzed by using FlowJo version 7.6.5 (Treestar).

Western blot analysis. Total proteins were extracted using lysis buffer (Pierce; Thermo Fisher Scientific, Inc.). The protein concentration was determined by bicinchoninic acid protein assay (Thermo Fisher Scientific, Inc.) and then denatured by heating the mixture of the protein and the loading buffer at 

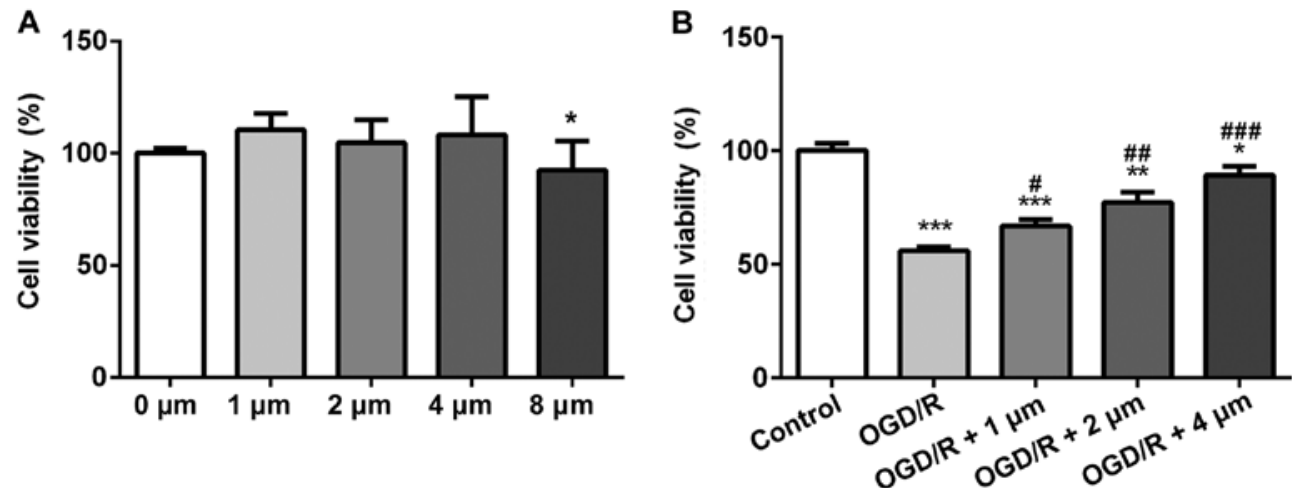

Figure 1. Butorphanol reduces cell viability following OGD/R injury. (A) The safe dose of butorphanol on PC12 cells was detected by CCK- 8 . " $\mathrm{P}<0.05$ vs. $0 \mu \mathrm{M}$. (B) Effect of butorphanol (1-4 $\mu \mathrm{M})$ on the viability of PC12 cells after OGD/R injury. Data are expressed as the mean \pm standard deviation (n=3). " $\mathrm{P}<0.05$, ${ }^{* *} \mathrm{P}<0.01$ and ${ }^{* * *} \mathrm{P}<0.001$ vs. Control; ${ }^{\#} \mathrm{P}<0.05,{ }^{\# \#} \mathrm{P}<0.01$ and ${ }^{\# \# \#} \mathrm{P}<0.001$ vs. OGD/R. OGD/R, oxygen glucose deprivation/reoxygenation.

$95^{\circ} \mathrm{C}$ for $5 \mathrm{~min}$. A total of $40 \mu \mathrm{g}$ protein per lane was resolved by $12.5 \%$ SDS-PAGE and transferred to a PVDF membrane. Membranes were blocked with $5 \%$ skimmed milk at $37^{\circ} \mathrm{C}$ for $2 \mathrm{~h}$ and incubated with primary antibodies against Bcl-2 $(1: 1,000)$, Bax $(1: 1,000)$, XIAP $(1: 1,000)$, cleaved-PARP $(1: 1,000)$, caspase-3 $(1: 1,000)$, caspase-9 $(1: 1,000)$, cleaved-caspase-3 and $-9(1: 1,000)$ overnight at $4{ }^{\circ} \mathrm{C}$. After washing the membrane with TBS with $0,1 \%$ Tween-20, the HRP-conjugated goat anti-rabbit secondary antibody $(1: 5,000)$ was added at room temperature for $1 \mathrm{~h}$, and signals were visualized using ECL reagent (Cytiva). The gel images were scanned by the Typhoon 9400 Gel Imaging System (Cytiva) and analyzed by Quantity One software (version 4.6.9; Bio-Rad Laboratories, Inc.). $\beta$-actin was used as the internal reference, and the relative expression levels of the target protein was expressed as the ratio of the gray value of target protein to $\beta$-actin.

Immunofluorescence. Cells were cultured on a 6-well plate at a density of $2 \times 10^{4}$ cells $/ \mathrm{ml}$. When the cell density grew to $80-90 \%$, the cells were digested using a trypsin-EDTA (0.05\% trypsin) solution and washed with PBS three times. Cells were fixed with $4 \%$ paraformaldehyde at $4^{\circ} \mathrm{C}$ for $30 \mathrm{~min}$ and permeabilized in $0.5 \%$ Triton X-100 for $20 \mathrm{~min}$ on ice. Blocking was performed by incubation with $5 \%$ goat serum (Zymed; Thermo Fisher Scientific, Inc.) at room temperature for $30 \mathrm{~min}$. Cells were incubated with anti-XIAP antibodies (1:200) at $4^{\circ} \mathrm{C}$ overnight and subsequently incubated with FITC-conjugated goat anti-rabbit IgG antibodies $(1: 2,000)$ in the dark at $4^{\circ} \mathrm{C}$ for $2 \mathrm{~h}$. The cell nuclei were stained using DAPI and incubated at room temperature for $10 \mathrm{~min}$. The experimental results were observed using an Olympus BX60 fluorescence microscope at a magnification of x400 (Olympus Corporation).

ATP activity analysis. Cells were seeded in 6-well plates at a density of $5 \times 10^{3}$ cells/200 $\mu$ l. After the incubation, the cells and the supernatants were collected. The supernatant was centrifuged at $12,000 \times \mathrm{g}$ for $5 \mathrm{~min}$ at $4^{\circ} \mathrm{C}$ after pyrolysis at $4^{\circ} \mathrm{C}$. The concentration of ATP was determined using an ATP detection kit according to the manufacturer's protocol.

Statistical analysis. All experiments were repeated three times, and the data are expressed as the mean \pm standard deviation.
The data were analyzed using SPSS 22.0 (IBM Corp.). The statistical comparisons among multiple groups were performed using ANOVA followed by a post hoc Tukey's test. $\mathrm{P}<0.05$ was considered to indicate a statistically significant difference.

\section{Results}

Butorphanol increases cell viability following $O G D / R$ injury. The effects of different concentrations of butorphanol on the viability of PC12 cells was assessed using a CCK-8 assay. Compared with the control group, butorphanol (1,2 and $4 \mu \mathrm{M})$ had no effect on cell vitality (Fig. 1A), whereas in cells treated with $8 \mu \mathrm{M}$ butorphanol, viability was decreased. Thus, the former three groups were selected for subsequent study. To determine the protective effects of butorphanol on neurons in OGD/R injury, cells were treated with hypoxia and reoxygenation, and then subsequently with butorphanol. As shown in Fig. 1B, cell viability in the OGD/R group decreased significantly compared with the control group. Whereas, butorphanol increased cell viability compared with the OGD/R group in a dose-dependent manner. Cell viability was highest in cells treated with $4 \mu \mathrm{M}$.

Effect of butorphanol on expression of inflammatory factors following $O G D / R$ injury. The inflammatory response following ischemic injury is accompanied by the production of a large number of inflammatory factors. Thus, ELISA kits were used to detect the expression of several typical inflammatory factors (TNF- $\alpha$, IL-1 $\beta$, IL-6 and MCP-1). As shown in Fig. 2, compared with the control group, the expression of inflammatory cytokines in the OGD/R-treated cells was significantly increased, particularly MCP-1 expression. Butorphanol significantly reduced the expression of inflammatory factors compared with the OGD/R group, and the inhibitory effect was negatively associated with concentration. In addition, there was no significant difference in the expression of IL-6 between the $4 \mu \mathrm{M}$ group and the blank control group.

Effect of butorphanol on oxidative stress in OGD/R injury. The effect of butorphanol on oxidative stress induced by OGD/R in PC12 cells was assessed. As shown in Fig. 3, the OGD/R group significantly increased the levels of ROS, the release of LDH and the MPO content compared with the control group. Treatment 


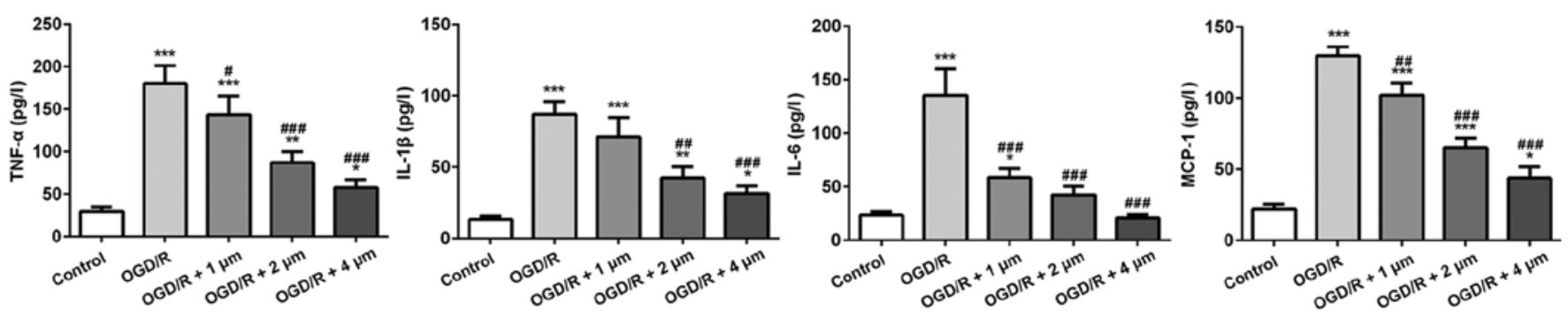

Figure 2. Effect of butorphanol on expression of inflammatory factors following OGD/R injury. ${ }^{*} \mathrm{P}<0.05,{ }^{* * *} \mathrm{P}<0.01$ and ${ }^{* * *} \mathrm{P}<0.001$ vs. control; ${ }^{~} \mathrm{P}<0.05,{ }^{\# \#} \mathrm{P}<0.01$ and ${ }^{\# \# /} \mathrm{P}<0.001$ vs. OGD/R. TNF- $\alpha$, tumor necrosis factor- $\alpha$; IL-, interleukin; MCP-1, monocyte chemoattractant protein-1; OGD/R, oxygen glucose deprivation/reoxygenation.
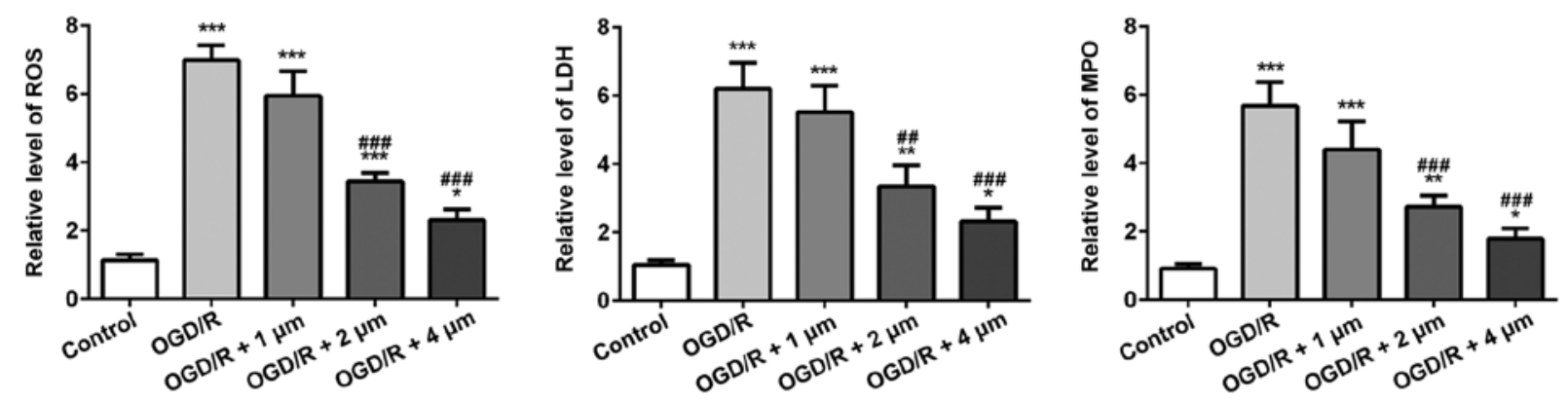

Figure 3. Effect of butorphanol on oxidative stress in OGD/R injury. ${ }^{*} \mathrm{P}<0.05,{ }^{* *} \mathrm{P}<0.01$ and ${ }^{* * *} \mathrm{P}<0.001$ vs. control; ${ }^{\# \#} \mathrm{P}<0.01$ and ${ }^{\# \# \#} \mathrm{P}<0.001$ vs. OGD/R. ROS, reactive oxygen species; LDH, lactate dehydrogenase; MPO, myeloperoxidase; OGD/R, oxygen glucose deprivation/reoxygenation.
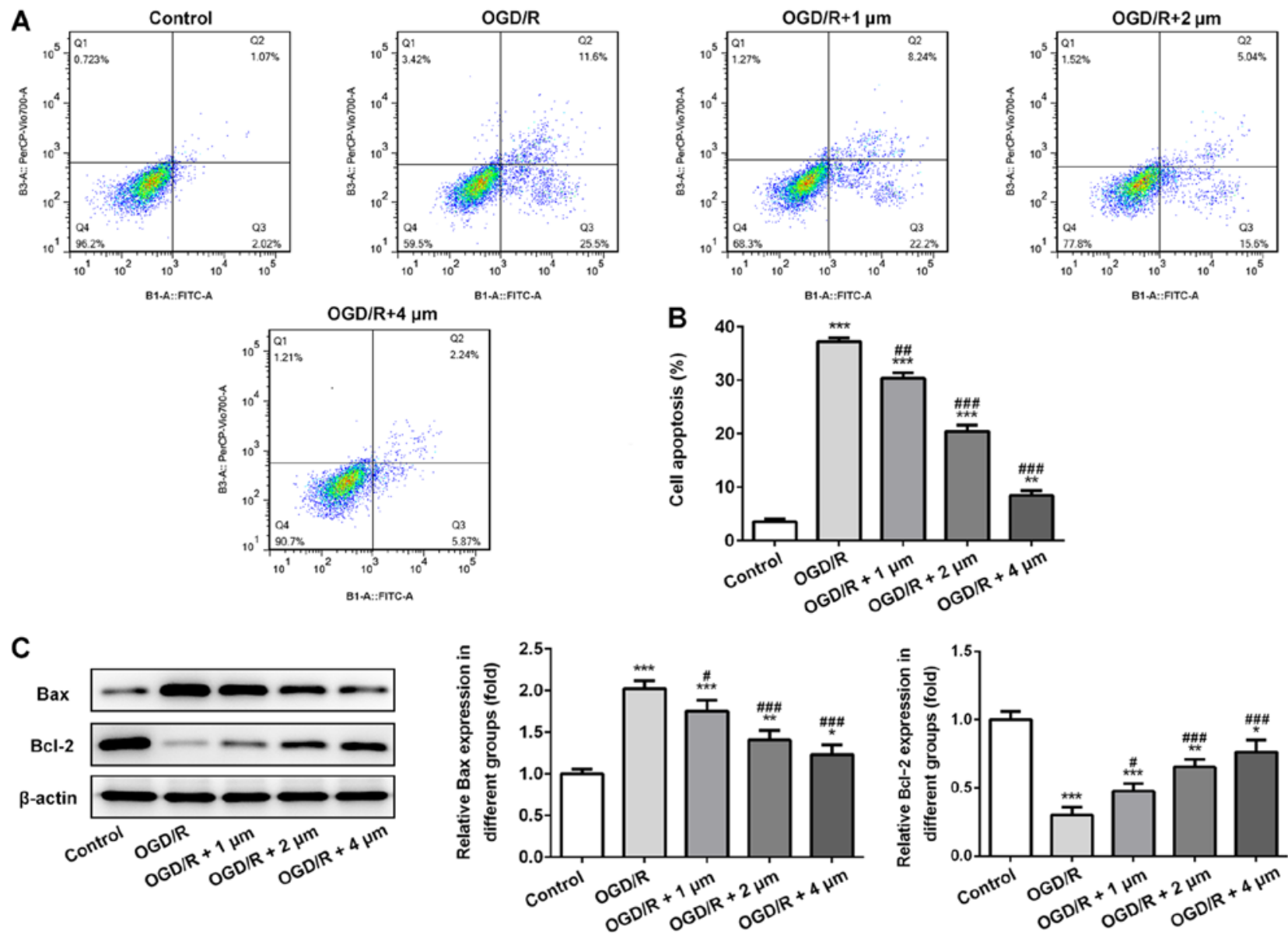

Figure 4. Effect of butorphanol on apoptosis of PC12 cells following OGD/R injury. (A and B) Apoptosis of PC12 cells in each group. (C) Relative protein levels of Bax and Bcl-2 in each group, $\beta$-actin was used as the control. ${ }^{*} \mathrm{P}<0.05,{ }^{* *} \mathrm{P}<0.01$ and ${ }^{* * *} \mathrm{P}<0.001$ vs. control; ${ }^{\#} \mathrm{P}<0.05,{ }^{\# \#} \mathrm{P}<0.01$ and ${ }^{\# \# *} \mathrm{P}<0.001$ vs. OGD/R. $\mathrm{OGD} / \mathrm{R}$, oxygen glucose deprivation/reoxygenation. 


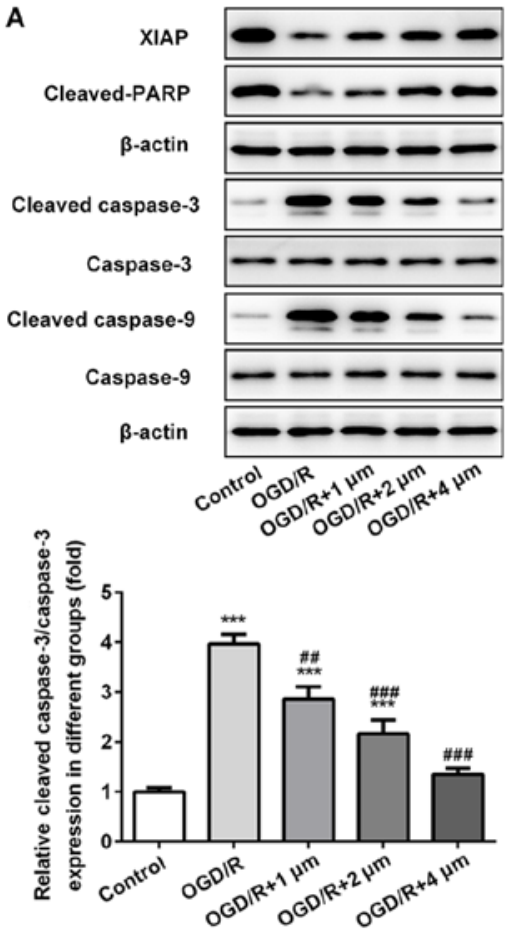

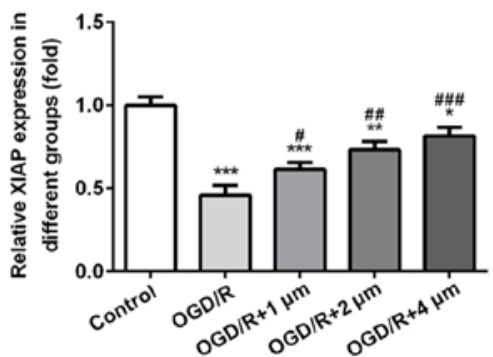
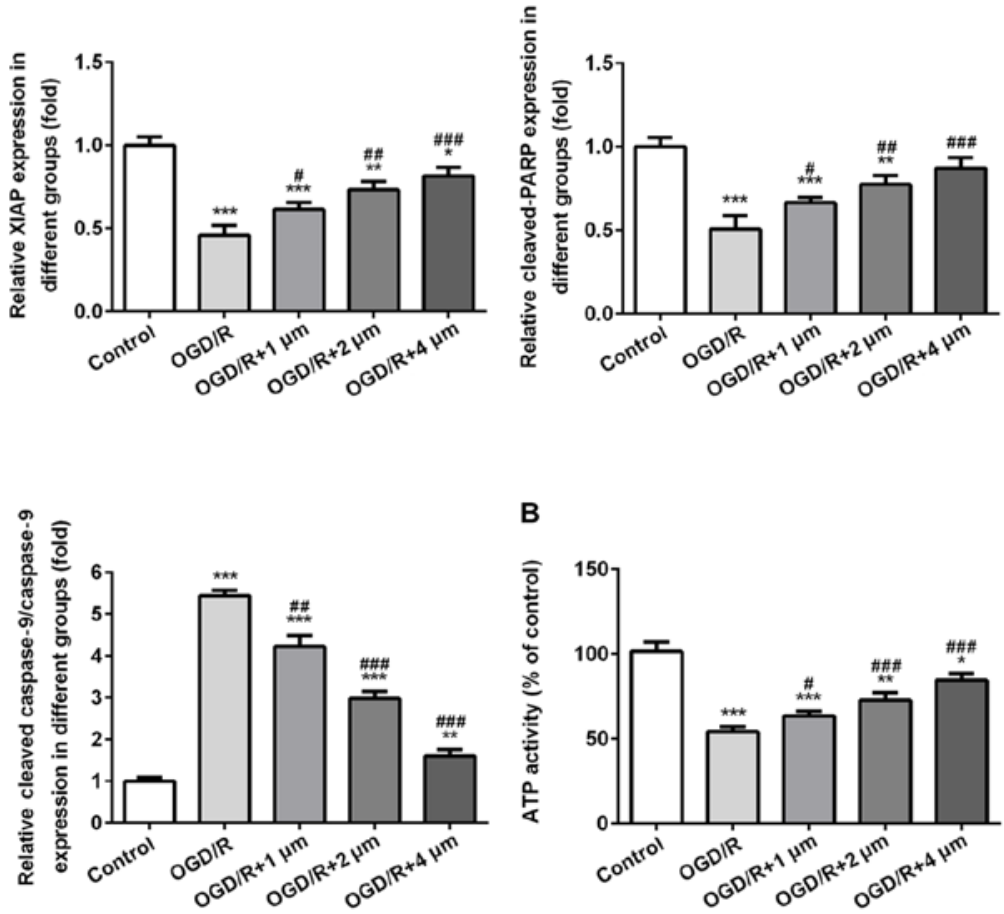

B

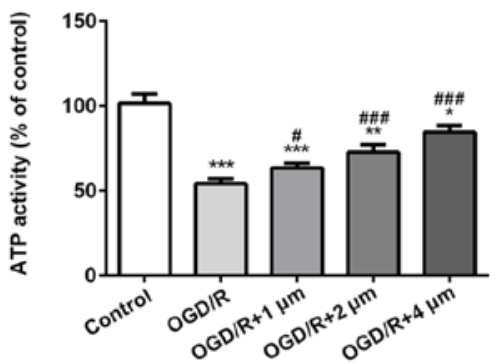

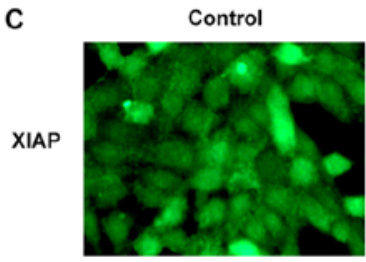
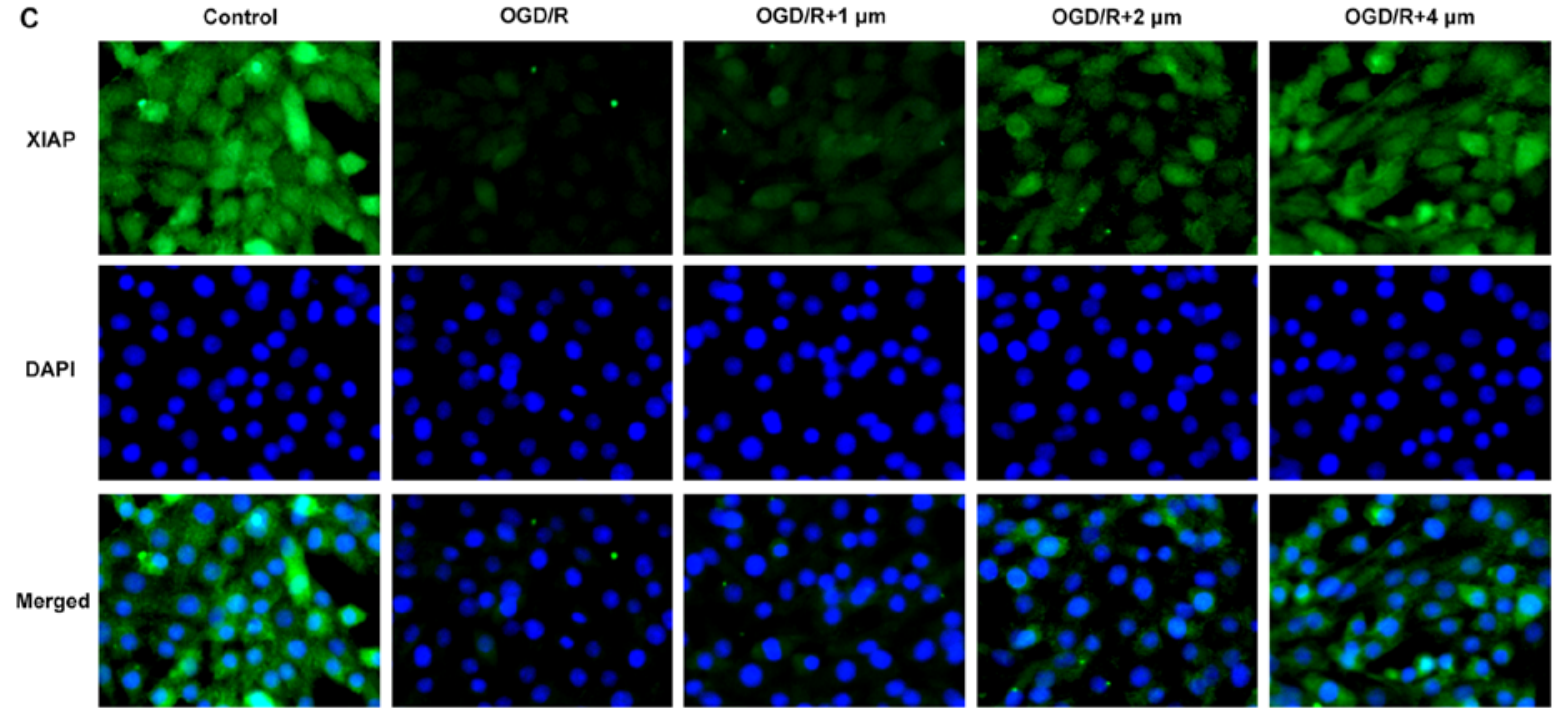

Figure 5. Effects of butorphanol on expression of mitochondrial-mediated apoptosis associated proteins and ATP activity. (A) Relative protein levels of XIAP, cleaved-PARP, caspase-3, caspase-9, cleaved caspase-3 and cleaved caspase-9 in each group, $\beta$-actin was used as the control. (B) ATP activity of PC12 cells was determined by a detection kit. (C) XIAP protein expression was examined by an immunofluorescence assay. ${ }^{*} \mathrm{P}<0.05,{ }^{* *} \mathrm{P}<0.01$ and ${ }^{* * * *} \mathrm{P}<0.001 \mathrm{vs}$. control; ${ }^{\#} \mathrm{P}<0.05,{ }^{\# \#} \mathrm{P}<0.01$ and ${ }^{\# \# \#} \mathrm{P}<0.001$ vs. OGD/R. OGD/R, oxygen glucose deprivation/reoxygenation; XIAP, X-linked inhibitor of apoptosis protein; PARP, poly ADP-ribose polymerase.

with butorphanol significantly decreased ROS, LDH and MPO levels, compared with the OGD/R group, in a dose-dependent manner. Consistent with the results of inflammatory factors, the inhibitory effect at $4 \mu \mathrm{M}$ was the most prominent.

Effect of butorphanol on apoptosis of PC12 cells following $O G D / R$ injury. Subsequently, apoptosis of PC12 cells was assessed by measuring the expression of the anti-apoptotic protein Bcl-2 and the pro-apoptotic protein Bax by western blotting. As shown in Fig. 4A and B, OGR/D significantly increased the apoptotic rate, and this was significantly alleviated by butorphanol. Similarly, as shown in Fig. 4C, compared with the control group, OGD/R significantly decreased the expression of Bcl-2 and increased the expression of Bax. Following treatment with butorphanol, there was a negative association between the expression levels of Bax and Bcl-2. The expression levels of Bax were decreased and the expression levels of Bcl-2 increased in a dose-dependent manner. Compared with the OGD/R group, there were significant differences between expression levels of Bax and Bcl-2 after butorphanol treatment.

Effects of butorphanol on expression of mitochondrial-mediated apoptosis associated protein and ATP activity. The expression of mitochondrial-mediated apoptosis associated proteins and ATP activity in PC12 cells induced by OGD/R 
were assessed. As shown in Fig. 5A and B, the expression levels of XIAP and cleaved-PARP was significantly decreased following OGD/R, whereas the expression of cleaved-caspase-3 and -9 was significantly increased. Following treatment with butorphanol, compared with the OGD/R group, butorphanol significantly increased the expression of XIAP and cleaved-PARP, and decreased the activation of caspase-3 and -9 . In addition, the activity of ATP in mitochondria was detected. Compared with the control group, OGD/R resulted in a significant reduction of ATP activity, and butorphanol increased activity in a dose-dependent manner. The expression levels of XIAP was also evaluated in $\mathrm{PC} 12$ cells treated with OGD/R by immunofluorescence. As shown in Fig. 5C, compared with the control group, OGD/R significantly reduced the expression levels of XIAP. Butorphanol increased the expression of XIAP in a dose-dependent manner.

\section{Discussion}

In the present study, the potential protective effects of butorphanol on nerve injury induced by OGD/R were assessed. To elucidate the effects of butorphanol on ischemia-reperfusion injury of PC12 cells, cell viability, expression of inflammatory factors, oxidative stress and apoptosis were measured. Butorphanol increased viability and decreased the intracellular levels of ROS, LDH and MPO in PC12 cells following OGD/R. Butorphanol also ameliorated the downregulation of $\mathrm{Bcl}-2$, the upregulation of Bax and the activation of mitochondrial apoptosis-related proteins. Additionally, butorphanol significantly enhanced ATP activity in the mitochondria.

A previous study demonstrated that butorphanol attenuated myocardial ischemia-reperfusion injury in mice by inhibiting mitochondrial-mediated apoptosis (16). In the present study, it was shown that butorphanol exhibited a protective effect on the activity of PC12 neurons against OGD/R-induced damage, consistent with previous studies.

There are a large number of inflammatory factors in the ischemic area, and the presence of inflammatory cells alters brain tissue from ischemia to inflammation by activating inflammatory signaling pathways during cerebral ischemia-reperfusion. Therefore, inflammation serves an important role during cerebral ischemia-reperfusion injury (21-23). In addition, butorphanol has been shown to exhibit anti-inflammatory effects. A previous study showed that butorphanol alleviated brain injury and neurological damage, and reduced the expression of serum inflammatory factors (TNF- $\alpha$, IL-6 and IL-1) by regulating the $\mathrm{NF}-\kappa \mathrm{B}$ signaling pathway in septic rats (24). In the present study, the expression of inflammatory cytokines in PC12 cells treated with OGD/R increased significantly, whereas butorphanol decreased the expression of inflammatory cytokines in a dose-dependent manner. Therefore, butorphanol can reduce OGD/R-induced neuronal injury by inhibiting the expression of inflammatory factors.

Oxidative stress serves a key role in neuronal injury following cerebral ischemia (25). Wu et al (18) found that butorphanol postconditioning significantly reduced MPO content in cardiomyocytes following ischemia injury. In the present study, the levels of ROS, LDH and MPO in the OGD/R group were all significantly increased, and addition of butorphanol reduced their levels. Therefore, it is hypothesized that butorphanol may protect PC12 cells from OGD/R-induced injury by inhibiting oxidative stress.

The causes and pathways underlying apoptosis are complex and diverse, and numerous genes are involved in the regulation of apoptosis, including lethal genes and survival genes. Among them, members of the Bcl-2 family serve an important role in gene regulation. They are primarily divided into anti-apoptotic gene Bcl-2 and pro-apoptotic gene Bax, which regulates apoptosis by activating a series of downstream genes $(26,27)$. In the present study, OGD/R significantly decreased the expression of $\mathrm{Bcl}-2$ and increased the expression of Bax. Following treatment with butorphanol, the expression of Bcl-2 was significantly increased and the expression of Bax was significantly decreased. These data suggested that butorphanol attenuated nerve injury induced by OGD/R, which may be associated with the inhibition of apoptosis.

The process of ischemia-reperfusion injury drastically alters the steady-state balance of mitochondria, reduces the production of ATP and induces apoptosis and necrosis $(28,29)$. In the present study, it was shown that following hypoxia, the expression levels of XIAP and cleaved-PARP were decreased significantly, whereas the expression levels of cleaved-caspase- 3 and -9 were increased significantly. Butorphanol significantly blocked the activation of caspase-3 and -9, and increased the expression of XIAP and cleaved-PARP. Additionally, compared with the OGD/R group, butorphanol also significantly enhanced ATP activity following mitochondrial injury. Based on the above results, it was demonstrated that butorphanol reduced OGD/R-induced neuronal injury by inhibiting mitochondrial apoptosis.

In summary, it was shown that butorphanol can be used to protect neuronal PC12 cells from OGD/R-induced damage, including inflammatory and apoptotic damage, whilst also reducing mitochondrial damage. However, the specific underlying mechanisms should be further studied. The present study expands the potential application of butorphanol and highlights a novel method for the treatment of nerve-related ischemia-reperfusion injury.

\section{Acknowledgements}

Not applicable.

\section{Funding}

No funding was received.

\section{Availability of data and materials}

All data generated or analyzed during this study are included in this published article.

\section{Authors' contributions}

ZY and LW designed the research. ZY, LW, YH and FW performed the experiments. ZY analyzed the data and wrote the paper. All authors read and approved the final manuscript.

\section{Ethics approval and consent to participate}

Not applicable. 


\section{Patient consent for publication}

Not applicable.

\section{Competing interests}

The authors declare that they have no competing interests.

\section{References}

1. Yitshak Sade M, Novack V, Ifergane G, Horev A, Kloog I: Air pollution and ischemic stroke among young adults. Stroke 46 : 3348-3353, 2105 .

2. Dong JY, Iso H, Kitamura A and Tamakoshi A; Japan Collaborative Cohort Study Group: Multivitamin use and risk of stroke mortality: The Japan collaborative cohort study. Stroke 46 $1167-1172,2015$

3. Kim JY, Kawabori M and Yenari MA: Innate inflammatory responses in stroke: Mechanisms and potential therapeutic targets. Curr Med Chem 21: 2076-2097, 2014.

4. Nordestgaard LT, Tybjærg-Hansen A, Nordestgaard BG and Frikke-Schmidt R: Loss-of-function mutation in ABCA1 and risk of Alzheimer's disease and cerebrovascular disease. Alzheimers Dement 11: 1430-1438, 2015.

5. Dai Y, Wang Z, Quan M, Lv Y, Li Y, Xin HB and Qian Y: Asiatic acid protests against myocardial ischemia/reperfusion injury via modulation of glycometabolism in rat cardiomyocyte. Drug Des Devel Ther 12: 3573-3582, 2018.

6. Aon MA, Cortassa S, Marbán E and O'Rourke B: Synchronized whole cell oscillations in mitochondrial metabolism triggered by a local release of reactive oxygen species in cardiac myocytes. J Biol Chem 278: 44735-44744, 2003.

7. Shah VK and Shalia KK: Reperfusing the myocardium - a damocles Sword. Indian Heart J 70: 433-438, 2018.

8. Suhail AS, Suhel P and Heena T: Melatonin and ischemic stroke: Mechanistic roles and action. Adv Pharmacol Pharm Sci 31: 1-8, 2015. [J].

9. Waseem M, Tabassum H and Parvez S: Melatonin modulates permeability transition pore and 5-hydroxydecanoate induced KATP channel inhibition in isolated brain mitochondria. Mitochondrion 31: 1-8, 2016.

10. Sun M, Izumi H, Shinoda Y and Fukunaga K: Neuroprotective effects of protein tyrosine phosphatase $1 \mathrm{~B}$ inhibitor on cerebral ischemia/reperfusion in mice. Brain Res 1694: 1-12, 2018.

11. Tsukamoto A, Iimuro M, Sato R, Yamazaki J and Inomata T: Effect of midazolam and butorphanol premedication on inhalant isoflurane anesthesia in mice. Exp Anim 64: 139-145, 2015.

12. Gupta A, Kaur S, Attri JP and Saini N: Comparative evaluation of ketamine - propofol, fentanyl - propofol and butorphanol-propofol on haemodynamics and laryngeal mask airway insertion conditions. J Anaesthesiol Clin Pharmacol 27: 74-78, 2011.

13. Du BX, Song ZM, Wang K, Zhang H, Xu FY, Zou Z and Shi XY: Butorphanol prevents morphine-induced pruritus without increasing pain and other side effects: A systematic review of randomized controlled trials. Can J Anaesth 60: 907-917, 2013.

14. Lee H, Naughton NN, Woods JH and Ko MC: Effects of butorphanol on morphine-induced itch and analgesia in primates. Anesthesiology 107: 478-485, 2007.
15. Kuzumaki N, Suzuki A, Narita M, Hosoya T, Nagasawa A, Imai S, Yamamizu K, Morita H, Nagase H, Okada Y, et al: Effect of $\kappa$-opioid receptor agonist on the growth of non-small cell lung cancer (NSCLC) cells. Br J Cancer 106: 1148-1152, 2012.

16. Huang LH, Li J, Gu JP, Qu MX, Yu J and Wang ZY: Butorphanol attenuates myocardial ischemia reperfusion injury through inhibiting mitochondria-mediated apoptosis in mice. Eur Rev Med Pharmacol Sci 22: 1819-1824, 2018.

17. Zhang XT, Jun L, Yang JP, et al: Fentanyl combined with butorphanol protects myocardial ischemia/reperfusion injury via $\kappa$-opioid receptor-mediated Nrf2-ARE signaling. Int J Clin Exp Med 9: 2500-2506, 2016.

18. Wu Y, Wan J, Zhen WZ, Chen LF, Zhan J, Ke JJ, Zhang ZZ and Wang YL: The effect of butorphanol postconditioning on myocardial ischaemia reperfusion injury in rats. Interact Cardiovasc Thorac Surg 18: 308-312, 2014. [J].

19. Liu X, Zhu X, Chen M, Ge Q, Shen Y and Pan S: Resveratrol protects PC12 cells against OGD/ R-induced apoptosis via the mitochondrial-mediated signaling pathway. Acta Biochim Biophys Sin (Shanghai) 48: 342-353, 2016.

20. Wu Y, Shang Y, Sun SG, Liu RG and Yang WQ: Protective effect of erythropoietin against 1-methyl-4-phenylpyridinium-induced neurodegenaration in PC12 cells. Neurosci Bull 23: 156-164, 2007.

21. Suzuki S, Tanaka K and Suzuki N: Ambivalent aspects of interleukin-6 in cerebral ischemia: Inflammatory versus neurotrophic aspects. J Cereb Blood Flow Metab 29: 464-479, 2009.

22. Maddahi A, Kruse LS, Chen QW and Edvinsson L: The role of tumor necrosis factor- $\alpha$ and TNF- $\alpha$ receptors in cerebral arteries following cerebral ischemia in rat. J Neuroinflammation 8: 107, 2011.

23. He ZJ, Huang ZT, Chen XT and Zou ZJ: Effects of matrix metalloproteinase 9 inhibition on the blood brain barrier and inflammation in rats following cardiopulmonary resuscitation. Chin Med J (Engl) 122: 2346-2351, 2009.

24. Meng J, Jiang SJ, Jiang D and Zhao Y: Butorphanol attenuates inflammation via targeting NF- $\kappa \mathrm{B}$ in septic rats with brain injury. Eur Rev Med Pharmacol Sci 23 (Suppl 3): 161-170, 2019.

25. Liu PK, Grossman RG, Hsu CY and Robertson CS: Ischemic injury and faulty gene transcripts in the brain. Trends Neurosci 24: 581-588, 2001.

26. Tsukahara S, Yamamoto S, Tin-Tin-Win-Shwe, Ahmed S, Kunugita N, Arashidani K and Fujimaki H: Inhalation of low-level formaldehyde increases the $\mathrm{Bcl}-2 / \mathrm{Bax}$ expression ratio in the hippocampus of immunologically sensitized mice. Neuroimmunomodulation 13: 63-68, 2006.

27. Prakasa Babu P, Yoshida Y, Su M, Segura M, Kawamura S and Yasui N: Immunohistochemical expression of Bcl-2, Bax and cytochrome $\mathrm{c}$ following focal cerebral ischemia and effect of hypothermia in rat. Neurosci Lett 291: 196-200, 2000.

28. Fantinelli JC, González Arbeláez LF, Pérez Núñez IA and Mosca SM: Protective effects of N-(2-mercaptopropionyl)-glycine against ischemia-reperfusion injury in hypertrophied hearts. Exp Mol Pathol 94: 277-284, 2013.

29. Penna C, Perrelli MG and Pagliaro P: Mitochondrial pathways, permeability transition pore, and redox signaling in cardioprotection: Therapeutic implications. Antioxid Redox Signal 18: $556-599,2013$.

This work is licensed under a Creative Commons Attribution-NonCommercial-NoDerivatives 4.0 International (CC BY-NC-ND 4.0) License. 